\title{
A Flexible Handoff Prioritization Scheme for Improved Quality of Service in Mobile Networks
}

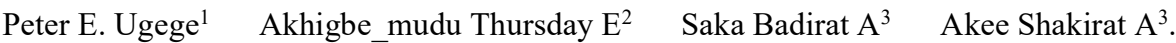 \\ 1.Department of Computer Science, Federal University of Agriculture, Abeokuta, Nigeria \\ 2.Babcock University, Ilishan-Remo, Nigeria \\ 3.Crescent University, Abeokuta Nigeria
}

\begin{abstract}
Efficient handoff prioritization schemes make mobile networks to perform better. However, most existing schemes have the common characteristics of reducing the call drop probability and increasing the call block probability; this decreases the general network performance. The reservation of channels for the use of handoff calls alone has not proved to be an efficient approach to optimally utilize the network limited channel resources. These approaches are such that even when the reserved channels are not in use, new calls could still get blocked if there are no channels among the shared channels to allocate to the new calls. In trying to maintain an improved QoS for handoff calls while ensuring an acceptable QoS for new calls, different methods have been suggested for new calls to access and use the reserved channels while priority is still given to handoff calls since it is more frustrating to have an on-going call dropped than blocking a new call. Network parameters such as call drop probability and traffic intensity had been used separately to determine when new calls could be granted access to use the reserved channels or otherwise. Handoff queuing and other call admission control approaches have also been used. This paper proposes the use of traffic intensity and call drop probability to decide whether the new calls could use the reserved channels or not. Also proposed is the need to keep the number of reserved channels fixed rather than calculating the numbers to reserve per time therefore reducing the computational complexity of the scheme. The simulation results showed an improved QoS for the handoff calls while producing a commensurate improvement for the new calls through a reduced Call drop Probability and call block probability.
\end{abstract}

Keywords: Call block probability, Call drop probability, traffic intensity, handoff prioritization, mobile network DOI: $10.7176 /$ JIEA/11-1-03

Publication date: January $31^{\text {st }} 2021$

\section{INTRODUCTION}

Handoff is the process that handovers an active call from cell to cell as mobile stations moves through the cellular system coverage area. Efficient handoff mechanism is extremely important because of the need to optimize channel utilization in mobile networks when a user switches between cells (Kaur and Kaur, 2014), (Goswani and Patel, 2014), (Solanki and Rafiq, 2014). The reason of the handoff procedure is to ensure active calls are preserved as users moves between cells (Nayak et al., 2015), (Goswani and Patel, 2014) and (Kar and Nayak, 2014). Dropping an active call frustrates a customer than blocking a new call. As a result handoff schemes prioritize handoff calls over new calls. Sometimes channels are kept idle waiting to be used for handoff calls even when new calls are being blocked for none availability of frequency resources to allocate for their use (Solanki and Rafiq, 2014), (Asuquo et al., 2014), (Kaur and Kaur. 2014). This has brought about the need for more efficient schemes that can adequately provide an acceptable QoS for both handoff calls and new calls in mobile networks. Such schemes will also reduce congestions in mobile networks.

Different approaches are suggested to ensure efficient handoff in mobile networks. An approach is to reduce the handoff failure rate by giving precedence to handoff calls above new calls (Khan, 2010). Basic methods in handoff prioritization schemes are call admission control (CAC), guard channels (GC) and handoff queuing schemes. Sometimes these schemes are combined together to obtain better results (Goswani and Patel, 2014). The most popular strategies for prioritizing handoff calls are the guard channel strategy and the handoff queuing strategy (Bilal et al, 2016). In guard channel schemes the chance of successful handoff is improved by keeping a number of channels for the exclusive use of handoff calls. The remaining channels are equally accessed by handoff and new calls. However this leads to a poor utilization of the scarce channel resources since guard channels are left unutilised at no-peak hours and yet inaccessible by new calls which are sometimes blocked for lack of channels to service them. Another issue with guard channels is the choice of the number of guard channels to reserve. According to (Bilal et al, 2016), a high guarded channels number leads to a very small value of call drop probability, but it will also lead to a very big value of call block probability (CBP) because more channels are reserved for handoffs which could lead to starvation of channels for new calls.

In the CAC schemes (Khan, 2010) a decision is made whether new call requests are allowed into the network or not. In the CAC some calls are blocked if the arrival rate of new calls is higher than a set threshold even if channels are available, this is to ensure a decrease in the probability of dropping a handoff call. In Queuing handoff call prioritization scheme (Khan, 2010), the handoff calls are queued when all channels are engaged until a channel 
is available to assign to the queued calls. These schemes often decrease the call dropping probability at the expense of a decreased call blocking probability. The proposed algorithm in this work provides an acceptable QoS for new calls even while the handoff calls are prioritized

\section{LITERTURE REVIEW}

The scheme proposed by (Bilal et al., 2016), combines guard channel reservation and handoff queuing to prioritize handoff calls. The results produced showed a clear improvement on the QoS of the handoff calls but to the detriment of that of new calls. There was a decrease in the call drop probability but the call block probability increased.

In the scheme proposed by (Ali et al., 2016), integer programming was employed to decide if a new call should be accepted or not. Information on the traffic intensity was used to decide at what point new call could be accepted. The scheme adopted a zero call dropping probability; meaning that once a call is accepted, it is never dropped before its normal completion as provision is made ahead for it to be serviced as it moves from cell to cell in the network. Though this brought a 100\% guaranteed service of handoff calls, it led to a high call block probability. Also a zero call drop probability is not realistic in a practical mobile network setting.

(Chavan and Sarasu, 2016) proposed a channel borrowing scheme in which the reserved channel for handoffs and other priority classified calls can be borrowed by new calls when idle. However the channel is withdrawn once a higher priority call lacks a channel to service it. The advantage is that it optimizes channel utilization since there is the possibility of the new call getting to use the channel all through without any handoff or higher priority call requesting for a channel. Beside if pre-empted it is kept on queue and considered to be serviced once a channel becomes available. The scheme used fuzzy logic control system. The scheme proved very effective under light traffic but as the traffic builds up, there is an increase in the call drop and call block probabilities.

(Nayak et al., 2015) proposed an adaptive channel assignment scheme that divides the network channels into guard channels and shared channels. They used Markovian model to estimate a threshold based on the traffic intensity. Depending on the threshold, the cells can be classified into two. The cell is cold when the number of channels used in the cell is less than the set threshold, else it is hot. A cold cell can admit both new and handoff calls while only handoff calls are accepted by hot cells. However, computing the quantity of channels to reserve for handoff calls all the time added to the computational time of this approach. Also they considered only one parameter in deciding when to reserve channels and how many channels to reserve for the handoff calls only.

(Kar and Nayak, 2014) presented an algorithm that is able to adjusts the number of guard channels dynamically depending on the handoff calls drop rate over a period of time. It keeps the handoff call rejection rate below the given threshold and it also reduces the new call rejection rate a bit by decrementing the guard channels number when it is observed to be more than needed.

(Anujendra and Thakurta, 2014) proposed a model that used a GA approach to optimize objectives such as number of channels and the quantity of blocked hosts. The proposed model was an effective approach to improve the network reliability by the proper management and efficient usage of the channel reuse factor.

(Solanki and Rafiq, 2014) proposed a scheme to improve the performance of new calls by using corresponding properties of the dual system that offers the benefit to share the traffic load with channel distribution methods amid the micro-macrocell. By using this approach, the load of the cell may be moved between two tiers (lower and upper tiers).

In (Susil and Prafulla, 2013), a hybrid channel assignment mechanism was introduced. The mechanism reduces the chance of call block and call drop by adopting hybrid frequency borrowing method and cell-based frequency distribution. They gave more importance to handoff calls. The simulation result shows a reduction in call drop and call block rates through disaster channel management implementation.

(Alagu and Meyyappau, 2012) presented a technique with adjustable guard channels that controls the number of guard channels reserved for handoff calls based on the traffic intensity.

\section{METHODOLOGY}

This paper proposes an enhanced handoff algorithm that is an improvement on existing works. Our improvements on existing works are centred on the following:

i. The algorithm uses two parameters (call drop probability and traffic intensity) to decide the accessibility to the fixed guard channels by new calls. Existing works by (Kar and Nayak, 2014) and (Alagu and Meyyappau, 2012) had used a single parameter

ii. Also the proposed algorithm gave special consideration to the handoff calls while ensuring as much as possible an acceptable QoS for new calls by not reserving channels for the exclusive use of handoff calls. (Nayak et al., 2015) gave special consideration to the handoff calls by reserving channels exclusively for handoff calls; although the number to reserve is calculated and varied per time. (Ali et al., 2016) proposed a zero call dropping probability (CDP).

iii. The algorithm kept the number of fixed guard channels constant and rather dynamically controls the 
access to the channels by new calls. The approach by (Nayak et al. 2015) had to calculate the number of channels to reserve each time.

The system architecture (Fig. 1), the proposed algorithm (Fig. 2) also represented with a flowchart (Fig. 3) considers the traffic intensity and blocking probability in the network. Both parameters are used to determine if access to the guard channels should exclusively be for handoff calls or otherwise. The algorithm therefore ensures that channels are not kept for handoff calls when it is not necessary to do so. This is intended to achieve more optimal utilization of the channel resources while maintaining a good QoS for both handoff and new calls.

The proposed algorithm permit new calls access to use the guard channels when the Traffic Intensity (TI) and/or the CDP are above the set Thresholds. A handoff call is only dropped under the condition that no guard channel is available and no channel could be assigned from the central pool. A neuro-genetic algorithm approach (Ugege and Ojesanmi, 2017) is employed to select the best channel to allocate from the central pool. The simulation was implemented using Matlab and the findings show that the algorithm guarantees a good QoS for both handoff and new calls.

\section{Parameters for the algorithm:}

- $\mathrm{TI}=$ traffic intensity $=($ Avail nom cha/Tot nom cha $)$

- $\mathrm{CBP}=$ call blocking probability $=\overline{\mathrm{NC}}$ rej/ Tot_N_calls

- $\mathrm{CDP}=$ call dropping probability $=\mathrm{HC}_{-}$rej/ Tot_H_calls

- H_calls = handoff/handoff calls

- N_calls $=$ New calls

- TI_threshold $=$ Traffic intensity threshold

- $\mathrm{CDP}$ threshold $=$ Call drop probability threshold

- HC_rej $=$ Handoff calls rejected

- NC_rej $=$ New calls rejected

- Tot_H_calls $=$ Total Handoff calls $=\mathrm{H}_{-}$calls $+\mathrm{HC}$ rej

- Tot_N_calls $=$ Total New calls $=$ N_calls + NC_rej

- Avail_nom_cha $=$ available nominal channel (Tot_nom_cha - Nom_cha_used)

_ Tot_nom_cha $=$ Total nominal channels

- Nom_cha_used $=$ Nominal channels in use

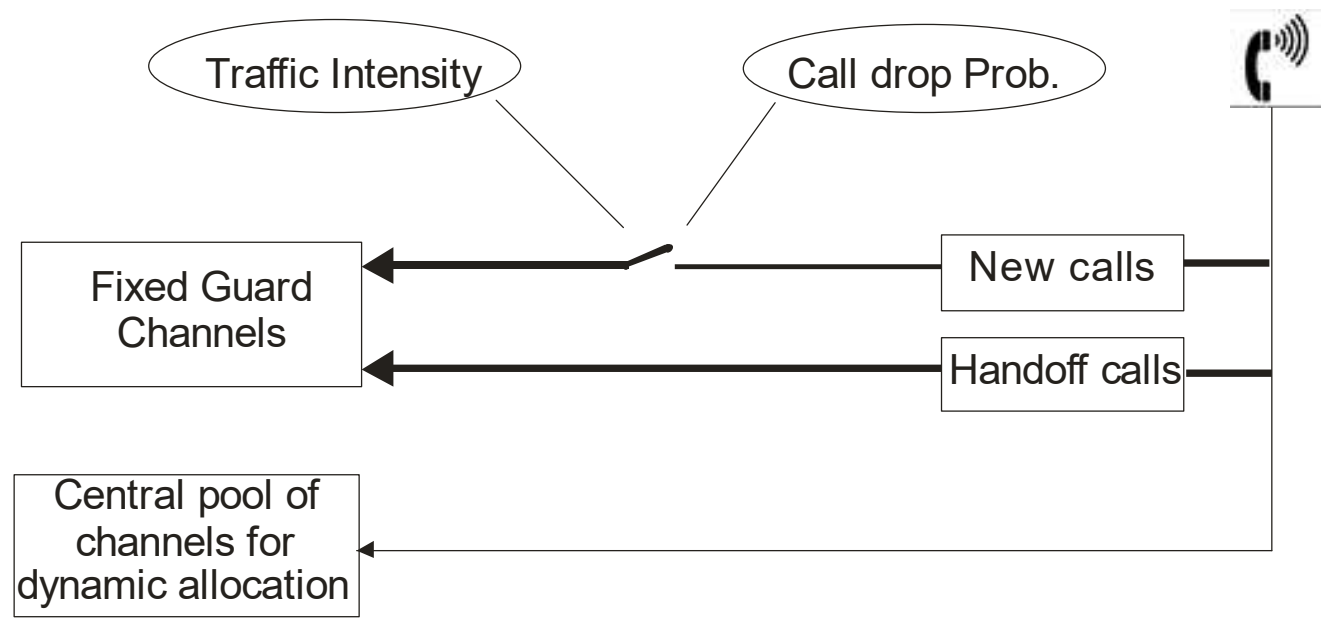

Fig. 1: The System architecture 


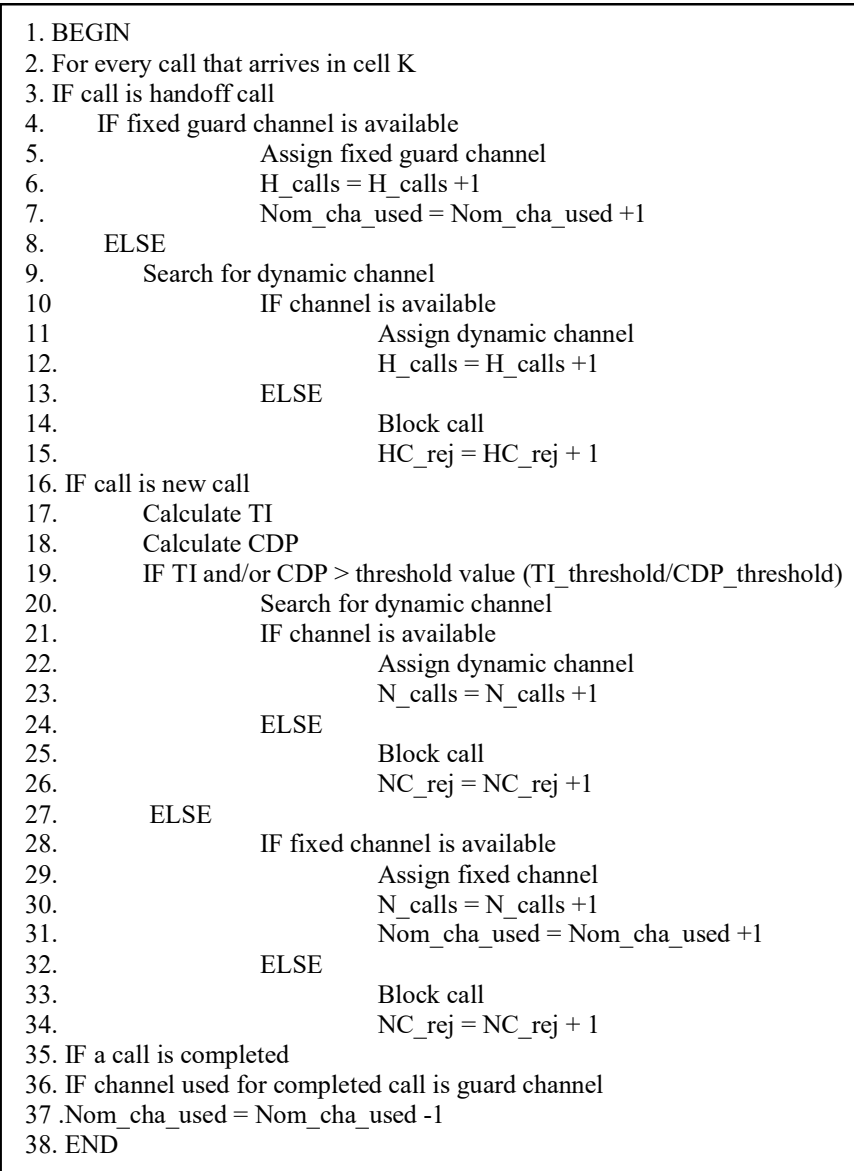

Fig. 2: Proposed Algorithm 


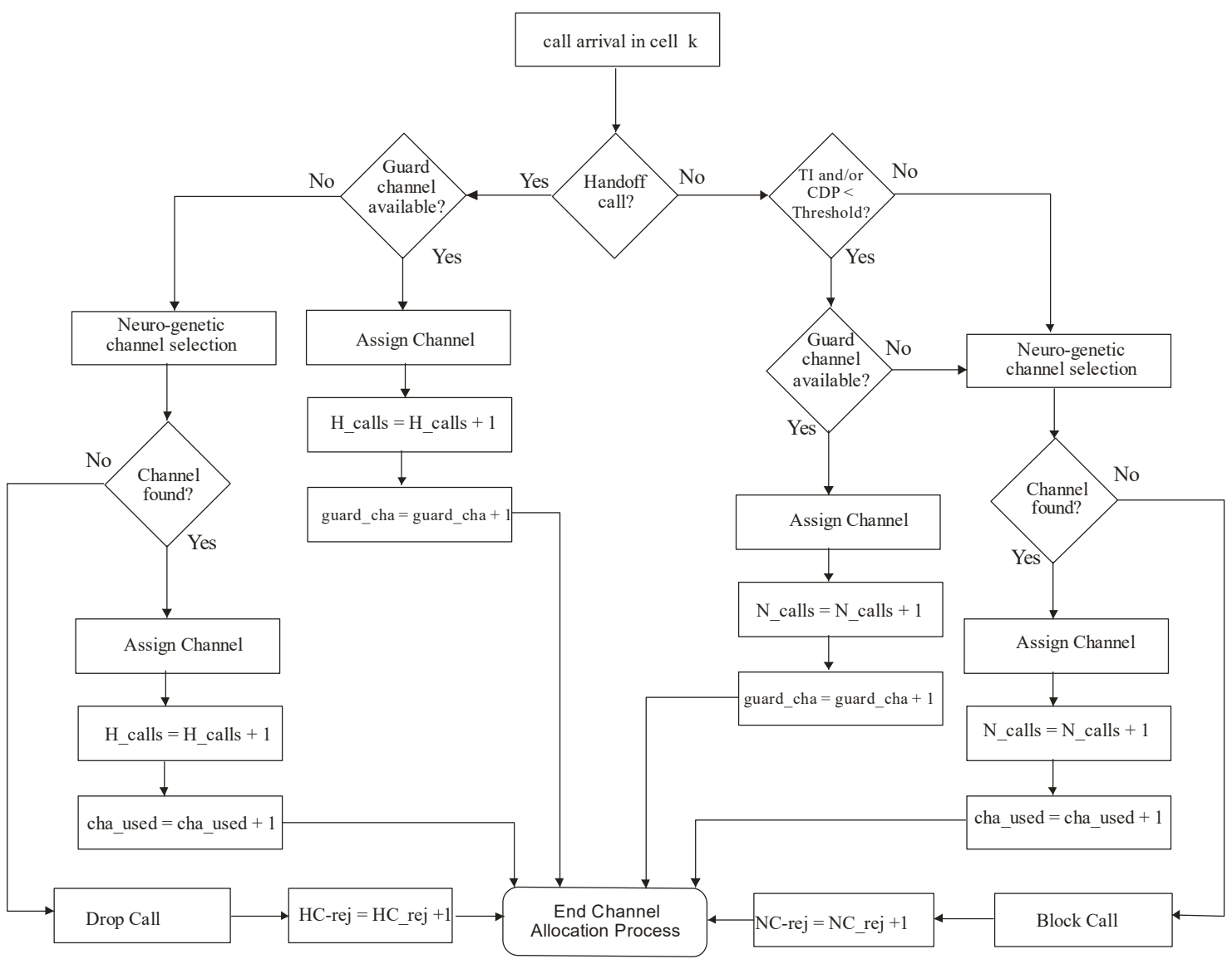

Fig. 3: Flowchart for the Algorithm

The results were benchmarked with existing schemes proposed by (Kar and Nayak, 2014) - KN2014, (Ali, 2016) - A2016, (Chavan and Sarasu, 2016) - CS2016 and data obtained from a leading mobile network service provider in Nigeria - NIGCDP.

\section{SIMULATION RESULTS}

Fig. 4 shows the mobile network simulation environment developed in MATLAB with mobile stations distribution across the network. Fig. 5 shows some handoff calls in red and Fig. 6 the distribution of channels to handoff calls only.

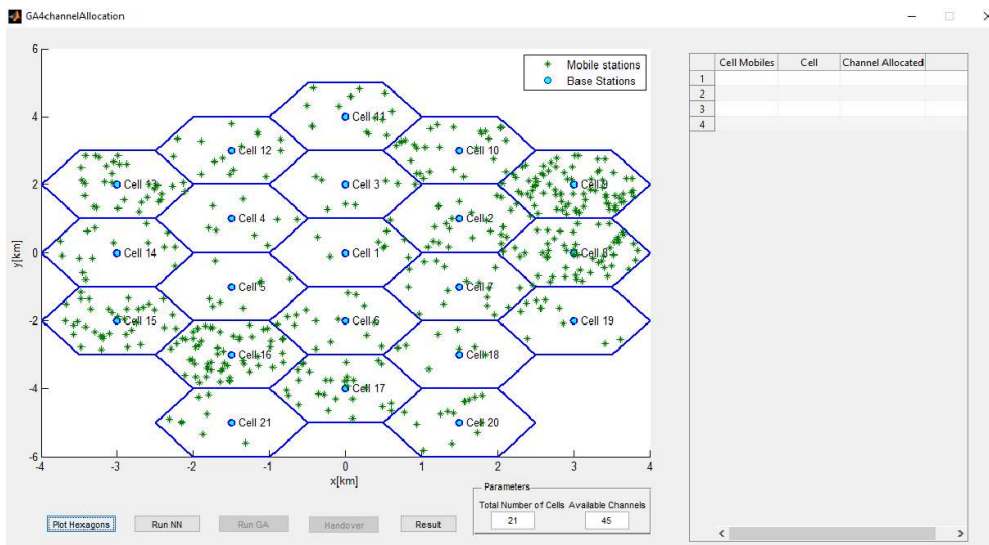

Fig. 4: Mobile network simulation environment developed with Matlab 


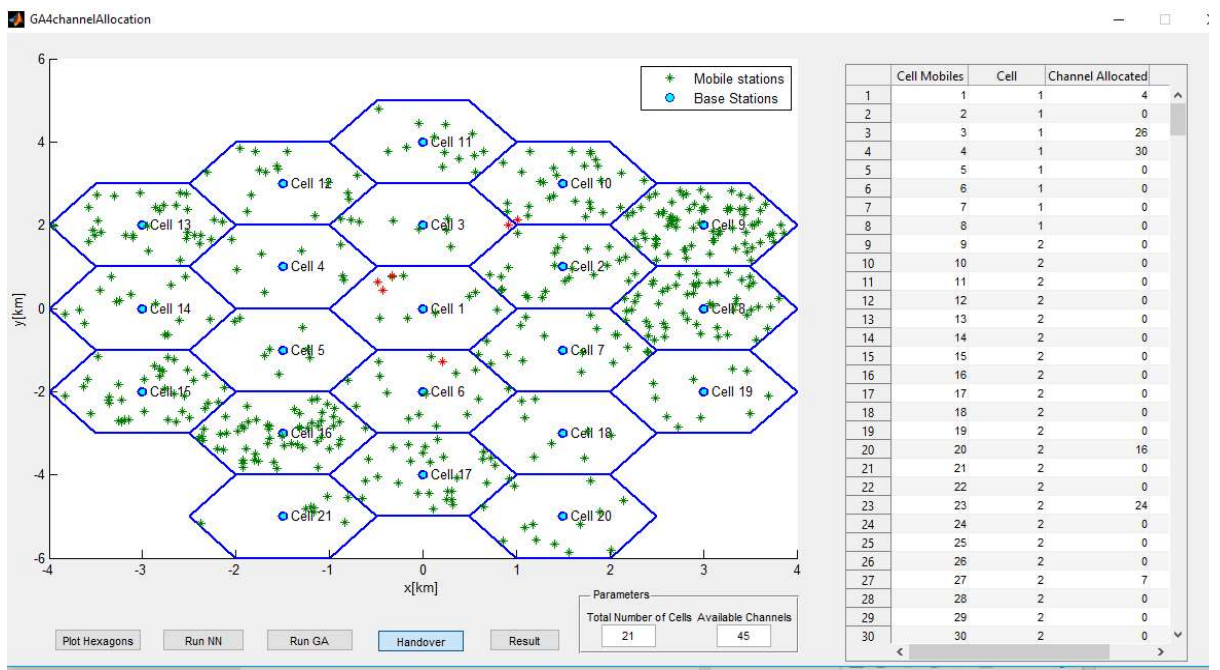

Fig. 5: Handoff calls in red and assigned channels to handoff calls

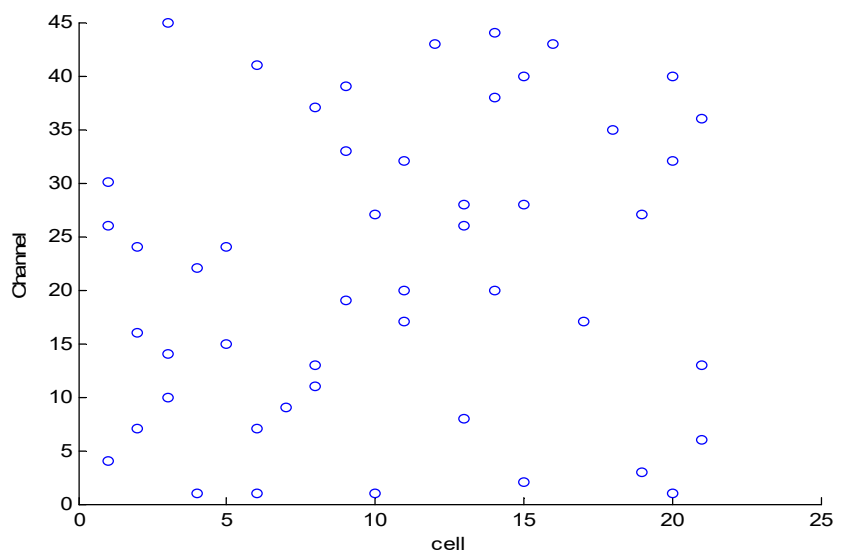

Fig. 6: Distribution of Channels assigned to handoff calls

From Fig. 7, the CDP of the proposed scheme is lower than that of other schemes at high traffic load with the exception of A2016 that had adopted an unrealistic zero CDP. The scheme also produced a lower CDP when compared to that of NIGCDP as shown in Fig. 8. NIGCDP is that of a leading mobile network provider in Nigeria. The CBP of CS2016 in Fig. 9 was slightly lower than that of the proposed scheme at traffic load of between 40 and 340 but beyond that, the proposed scheme performed better than other schemes. CS2016 at low traffic load allows new calls to use any available guard channel but these channels becomes unavailable as the traffic builds up and handoff and other priority calls increases. From the results, the proposed scheme performed better than other schemes at peak hours. The proposed scheme had produced both lower CDP and CBP at high traffic load when efficient handoff prioritization is required to avoid congestion. 


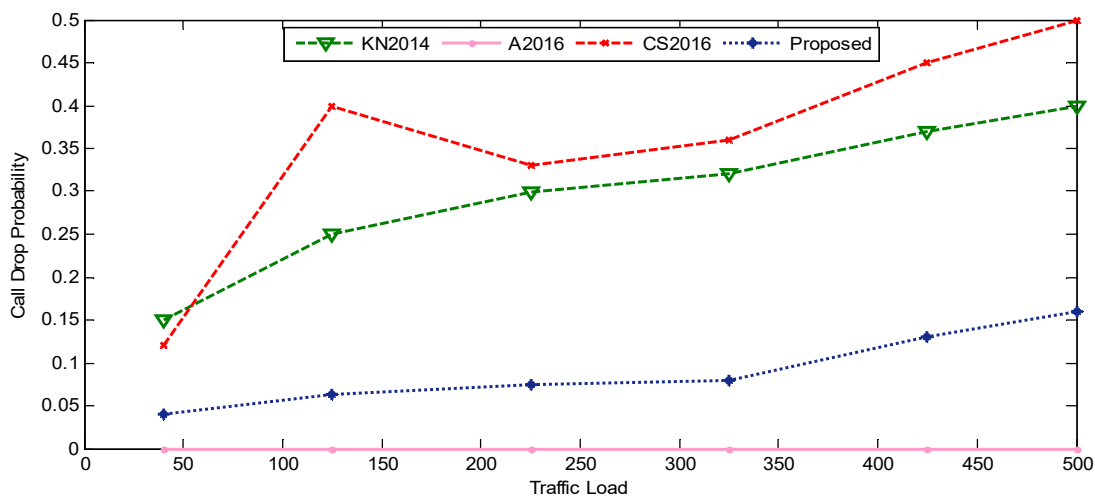

Fig. 7: CDP of existing schemes and proposed scheme

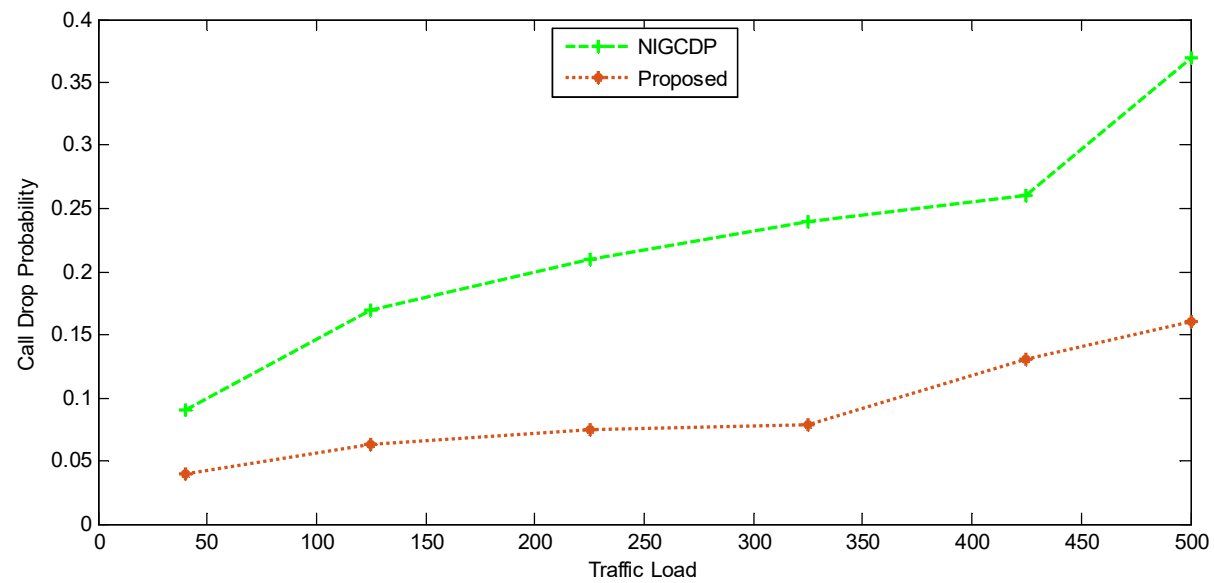

Fig.8: CDP of proposed scheme and a Leading Nigerian Mobile network service provider

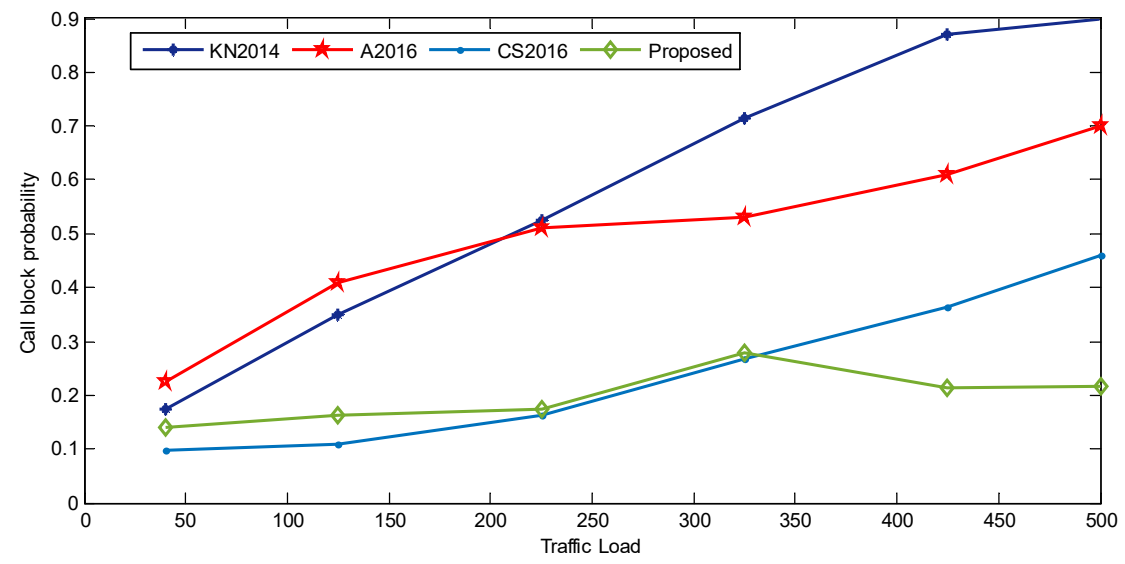

Fig. 9: CBP of existing schemes and proposed scheme

\section{CONCLUSION}

In this paper, a handoff prioritization scheme that uses values of the network traffic intensity and call drop probability to decide new calls admittance and access to reserved guard channels or otherwise is proposed. The scheme used a combined neural network and genetic algorithm approach to select the best channel to be assigned from the central pool when guard channels are not available. The primary aim of the scheme was to remove idleness 
of channels reserved for handoff calls while new calls are being blocked for lack of channels to service them. Reserved guard channels were only kept solely for the use of handoff calls only when the call drop probability or/and the traffic intensity of the network exceeds the set threshold value. The simulation results showed a better performance by the scheme at high traffic demands with lower call drop probability and call block probability. The scheme also kept the number of reserved guard channels fixed and rather dynamically controls access to the guard channels by new calls. The lower call drop and call block probabilities from the simulation results is a clear prove of a better optimal channel utilization by the scheme thus improving the QoS for both new and handoff calls.

\section{REFERENCES}

Alagu S. and Meyyappau T. 2012. Dynamic Channel Allocation Scheme to Handle Handoff in Wireless Mobile Network. Computer Science and Information Technology (CS \& IT), $171-184$

Ali A. 2016. Optimal Bandwidth Allocation with Bandwidth Reservation and Adaptation in Wireless Communication Networks. International Journal of Computer Networks and Communications (IJCNC) Vol 8 No. 1 January 2016, $21-33$

Anujendra A. and Thakurta P. K. G 2014. A New Reliability Based Channel Allocation Model in Mobile Networks. International Journal of Computer, Information Science and Engineering Vol. 8, No. 2, 265 - 269

Asuquo D. E, Williams E. E. and Nwachukwu E. O. 2014. A survey of Call Admission Control Schemes in Wireless Cellular Network. International Journal of Scientific and Engineering Research. 5(2): 111 - 120

Bilal O., Rola K and Hamza I. 2016. A New Handoff Strategy for Better Cellular network Performance. International Journal of Enhanced Research in Science, Technology and Engineering. Vol 5 Issue 2 February $2016,1-14$.

Chavan T. A. and Sarasu. P 2016. Channel Optimization Scheme for Call Admission Control Using Fuzzy Logic in Next Generation Wireless Networks. International Journal of Computer Science and Network. Volume 5 Issue 6. December 2016, 918 - 923

Goswani A. M. and Patel S. 2014. Ensuring better QoS through Call Admission Control in Wireless Network. International Journal of Computer Science and Information Technologies, Vol. 5(2), 1086 - 1089

Kaur H. and Kaur A. 2014. Various Handover Management Techniques in GSM Cellular System. International Journal for Technological Research in Engineering. Volume 1 Issue 11, 1335 - 1338

Khan J. 2010, Handover Management in GSM Cellular System International Journal of Computer Application. Volume 8- No.12, 37 - 42

Kar R. R. and Nayak S. S. 2014. An Efficient Adaptive Channel Allocation Scheme for Cellular Network. IOSR Journal of Computer Engineering (IOSR-JCE), Volume 16, Issue 2, 75-79

Nayak S. S., Kar R. R. and Garanayak M. 2015. Adaptive Shared Channel Assignment Scheme for Cellular Network to Improve the Quality of the Handoff Calls. IOSR Journal of Computer Engineering (IOSR-JCE) Volume 17 Issue 1, Ver. V. $61-67$

Solanki V. and Rafiq M. Q. 2014. Improving the Efficiency of Call Admission Control in Wireless Cellular Communication Network by Frequency Sharing Technology. International Journal of Computer Trends and Technology, Volume 9, Number 3, 133 -146

Susil K. S. and Prafulla K. B. 2013 "An Efficient Hybrid Channel Allocation Mechanism in Mobile Network" International Journal of Research in Engineering \& Applied Sciences Volume 3, Issue 22013.

Ugege P. E. and Ojesanmi O. A. 2017 “A Neural Network and Genetic Algorithm Scheme for Optimal Dynamic Channel Assignment in Mobile Networks" Proceedings of IEEE 3rd International Conference on ElectroTechnology for National Development, 7 - 10 November, 2017, FUTO Owerri. Pp 139 - 143 